\title{
Diffusion Controlled Oxidative Degradation of Un-stabilised Polyethylene
}

\author{
Drahosh Vesely ${ }^{*}$, Lucia Castro-Diaz \\ Department of Materials, Oxford University, UK
}

Copyright $\bigcirc 2016$ by authors, all rights reserved. Authors agree that this article remains permanently open access under the terms of the Creative Commons Attribution License 4.0 International License

\begin{abstract}
The chemical degradation processes of HDPE at $110^{\circ} \mathrm{C}$ in air have been studied using light microscopy, FTIR analysis, micro-hardness and $\mathrm{OsO}_{4}$ staining. It is shown that carbonyl groups $\mathrm{C}=\mathrm{O}$, (as detected by FTIR) are formed only in the surface layer and the effect on mechanical properties is marginal. On the other hand the formation of isolated and conjugated $\mathrm{C}=\mathrm{C}$ double bonds (as detected by $\mathrm{OsO}_{4}$ staining), show that cutting properties and micro-hardness are significantly altered. The progress of the degraded layer follows a diffusion process with sharp front and saturated concentration behind. An attempt is made to explain these observations by formation and mobility of free radicals. It is also shown that processing method, injection or compression moulding, can significantly influence the degradation process and the mechanical performance of the moulded component.
\end{abstract}

Keywords Permeability, Diffusion, uPVC, Acetone, FTIR Microscopy

\section{Introduction}

Degradation is a process that alters the chemical structure of the polymer, leading to deterioration of its physical properties [1]. The main reactions occurring in the process are cross linking, chain scission, double bonds formation and incorporation of oxygenated groups.

The degradation rate of $\mathrm{PE}$ at room temperature is very slow and experiments lasting 10-15 years are not practical. For this reason, studies of degradation are performed at elevated temperatures, on un-stabilised polymers and in oxygen environment. The chemical changes are then extrapolated to conditions of practical applications. The major effects of degradation are discolouration, changes in chemical structure and physical properties [2, 3, 4], particularly brittleness.

The importance of degradation for polymer application is shown by a substantial amount of literature available (e.g. [5-22]). As for many chemical reactions, degradation is based on initiation, propagation and termination of radicals, resulting in formation of more stable structures. Most reactions are dependent on the presence of oxygen and extraction of hydrogen. Solubility of oxygen in HDPE is sufficient for reactions, even at room temperature. Major functional groups formed are alkyl, alkoxy or peroxy radicals. Hydroperoxide are also formed and can decompose to hydroxy and peroxy radicals. Tertiary carbons are more reactive than secondary carbons and therefore have a shorter lifetime. Conjugated carbons are more stable than carbonyls and thus it can be expected that they dominate. Cross-linking also occurs and it will increase the strength but also the brittleness of the polymer. The number of possible reactions is very large and it is necessary to select those which are most common and result in more stable structures.

The degradation process is most often studied using FTIR spectroscopy considering the formation of carbonyls $(\mathrm{C}=\mathrm{O})$ as the major chemical reaction. However the FTIR analysis indicate that most carbonyls are formed within the surface layer, exponentially decreasing with depth, leaving the major part of the polymer thickness un-degraded. This can be explained by photo-oxidation $[17,18]$.

In the present work oxidation of HDPE is studied by comparing FTIR results with changes in mechanical properties, such as micro-indentation and cutting properties which, together with osmium tetroxide staining, reveal the concentration and progress of $\mathrm{C}=\mathrm{C}$ double bonds. Injection moulded samples as well as compression moulded samples, (which have more homogeneous structure) are used. This shows the importance of polymer processing methods on the performance and durability of polymer components.

\section{Experimental Methods}

Un-stabilised high density PE from BP (BP 5120) was injection moulded at Wolfson Centre for Polymer Processing, Brunel University, London, to form plates $147 \times 147 \times 4 \mathrm{~mm}$ and also tensile bars. Some samples were compression moulded from un-stabilised PE powder, using a laboratory press at compression force of 2 tons and temperature $170^{\circ} \mathrm{C}$. The formed discs were $65 \mathrm{~mm}$ in diameter and $3 \mathrm{~mm}$ thick. 
Samples were exposed to $110^{\circ} \mathrm{C}$ in air for different length of time from few hours to 265 days. The temperature in the oven was controlled to $\pm 0.5^{\circ} \mathrm{C}$.

Perkin Elmer Spectrum 2000 Explorer FTIR spectrometer with microscope was used to provide spatial variations in carbonyl absorption peak from selected area of $10 \times 400 \mu \mathrm{m}$, which provided spatial resolution of $10 \mu \mathrm{m}$. The integrated area of the peak can be plotted as carbonyl index $\mathrm{A}_{1716} / \mathrm{A}_{1492}$ (areas of peaks at $1716 \mathrm{~cm}^{-1}$ and $1492 \mathrm{~cm}^{-1}$ ). This technique provides a useful insight into the chemical structure of polymers [23-26]. However it does not reveal all the functional groups and it does not provide reliable quantitative information. Thin sections for FTIR were prepared using a microtome with metal or glass knife.

Cut thin samples as well as cut surfaces on bulk samples were investigated using transmission and reflection light microscopy. Some samples were exposed to osmium tetroxide vapour, using a specially constructed cell for safety [27]. It has been shown [28] that only isolated $\mathrm{C}=\mathrm{C}$ double bonds (formed by electron irradiation) could be stained. This is also discussed in chemistry textbooks (e.g. [29])

Micro-hardness was measured using Vickers diamond pyramidal probe on Matsuzawa MHT1 indenter. Pressure of $50 \mathrm{~g}$ was applied for $10 \mathrm{sec}$, and the probe was oriented with edges parallel and perpendicular to the sample edge. The Micro Hardness Vickers value was calculated as:

$$
\mathrm{MHV}=\mathrm{KP} / \mathrm{d}^{2}
$$

where constant $\mathrm{K}=18544$, applied load $\mathrm{P}$ in grams, diagonal $\mathrm{d}$ in microns and MHV in mega pascals [30]. Surface of polymer samples as well as cross sections were investigated and plotted as $\mathrm{H}-\mathrm{H}_{0}$, where $\mathrm{H}_{0}$ is the micro-hardness of un-degraded polymer.

\section{Experimental Results}

\subsection{Morphology of Un-degraded Samples}

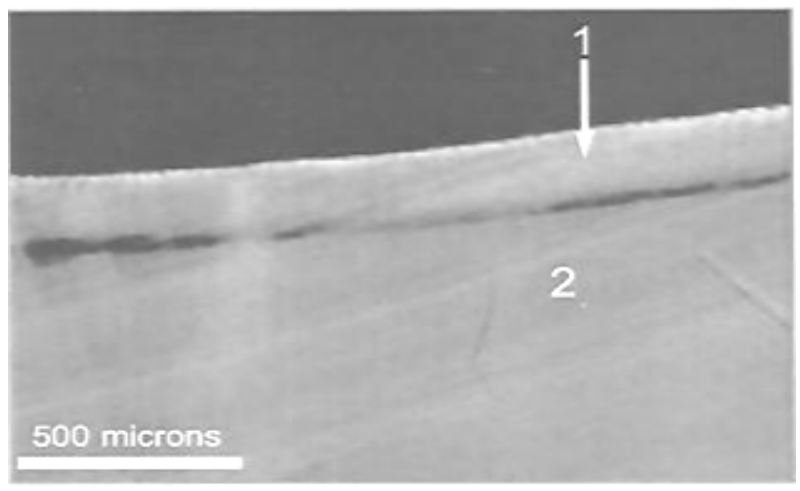

Figure 1. Transmission micrograph of a thin section (10-15 $\mu \mathrm{m}$ thick) of un-degraded polymer. The de-bonding of the skin 1 from the bulk 2 is clearly visible.
The only in-homogeneity observed by light microscopy on cut, injection moulded, un-degraded samples was the presence of the skin, 200-300 $\mu \mathrm{m}$ thick (see Figure 1). The skin is partially de-bonded due to stresses induced by cutting. Samples used for degradation studies had the skin removed from the moulded plate. The skin is most likely formed by rapid cooling of molten polymer in contact with the mould and subsequent injection of molten polymer. Compression moulded samples did not show any in-homogeneity.

\subsection{Degradation Layer}

The bulk samples were cut in half and investigated in reflection (Figure 2). Thin sections, about 10-20 microns thick, were also cut using a microtome and investigated in transmission (Figure 3a, 3b). The contrast in polarised light clearly visualised the degraded layer. Both types of samples indicate that the cutting properties are very similar throughout the degraded layer. It also reveals that there are cracks present. The degradation rate around the cracks follows the same degradation rate, showing that the cracks were partially present in the samples before degradation. The cracks are present in both injection moulded samples, i.e. in plates as well as in the tensile bars, but the compression moulded sample had no visible cracks and the degradation front is moving more slowly. The opening of the cracks also indicates shrinkage of the polymer by degradation. The tensile bars show $10 \%$ shrinkage in length after degradation. The crystallinity (as measured by DSC) has also increased about $10 \%$. However these changes are occurring simultaneously in the whole volume and therefore are not associated with the degraded layer.

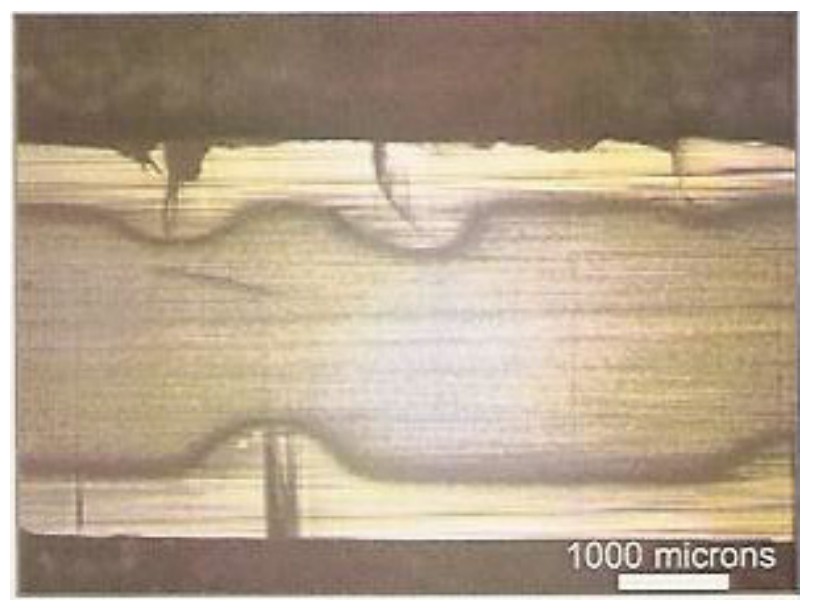

Figure 2. Cut surface of injection moulded specimen degraded for 10 days at $110^{\circ} \mathrm{C}$. Presence of cracks is accelerating degradation. A sharp boundary between degraded and un-degraded regions is clearly visible. Contrast between the regions was formed by different cutting properties. 

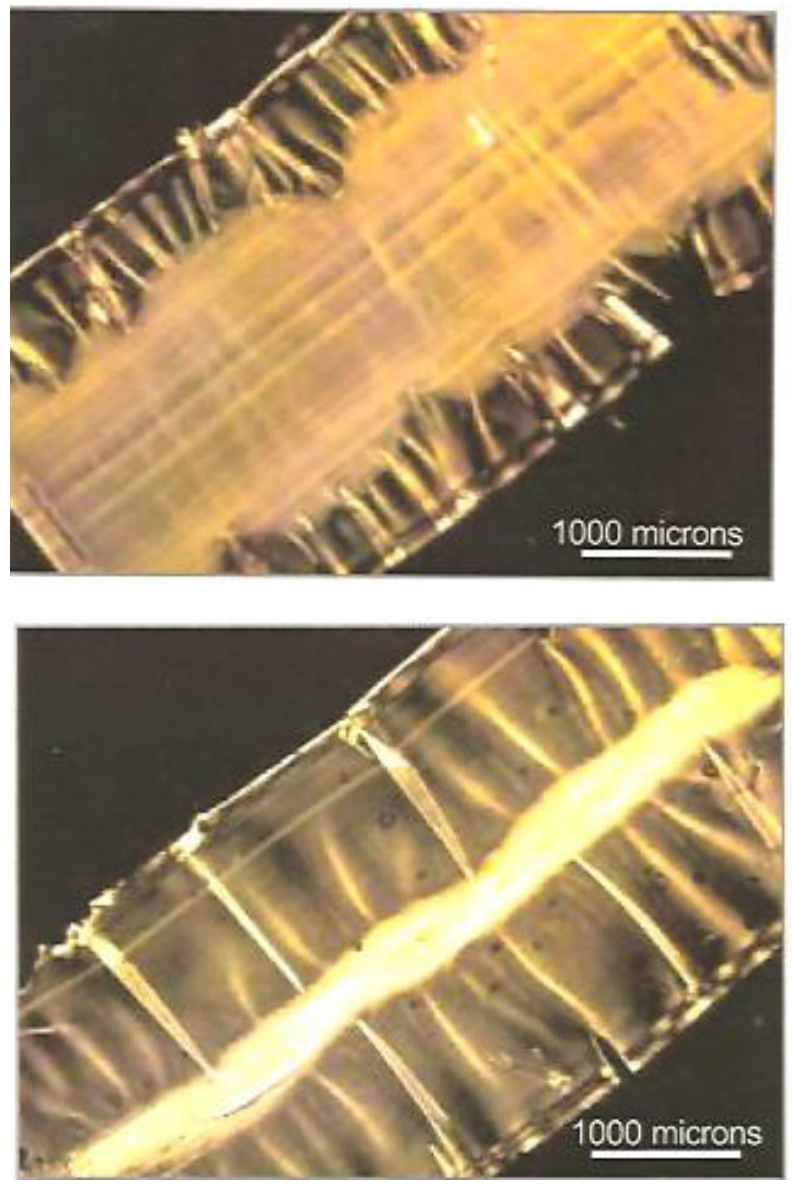

Figure 3a and 3b. Transmission polarized light microscopy of 10 microns thick cut section from samples degraded for 14 and 56 days respectively. Sharp contrast between degraded and non degraded core is clearly visible. The front progress can be accurately measured.

The progress of the degradation front with time can be measured and is plotted in Figure 4. The data are also fitted with the diffusion equation (2) [31, 32, 33].

$$
\mathrm{x}=\left(\mathrm{B}^{2}+2 \mathrm{At}\right)^{0.5}-\mathrm{B}
$$

where $\mathrm{B}$ is the initial rate limiting constant and $\mathrm{A}$ is the rate constant of the front progress. This equation has been derived on a simple assumption that the initial rate must be finite. For the best fit to experimental data the values of $\mathrm{B}=113$ microns and $\mathrm{A}=20000$ microns $^{2} /$ day have been used for injection moulded samples. For compression moulded samples the values $\mathrm{B}=157$ microns and $\mathrm{A}=9900$ microns ${ }^{2} /$ day were used. This indicates that the degradation front moves twice as fast for injection moulded sample than for the compression moulded sample. This can be explained by the presence of surface defects.

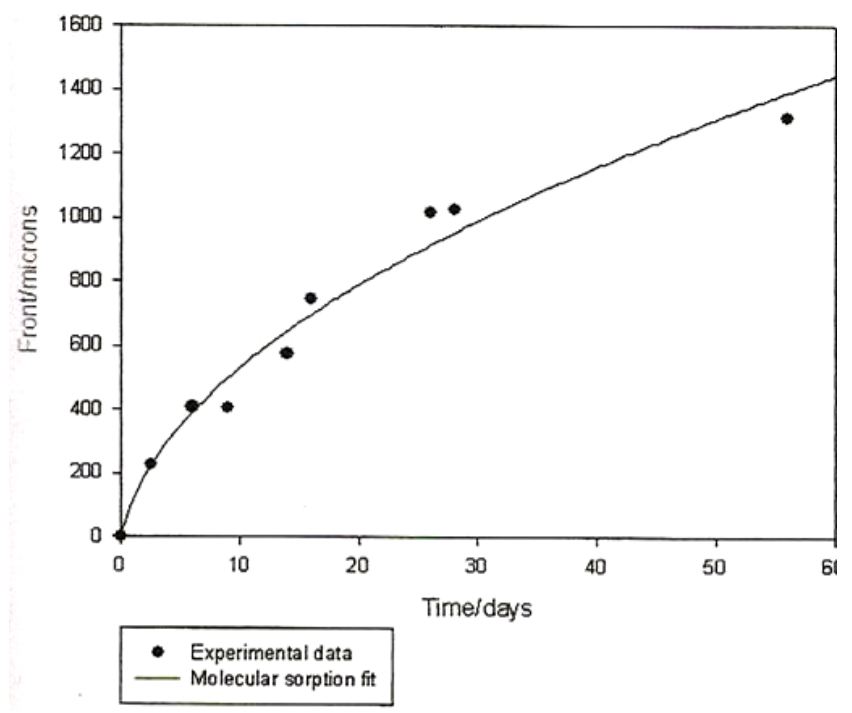

Figure 4. Progress of the degradation boundary with time is plotted. Data are fitted with the equation (2). The scatter of the data is much influenced by the presence of the surface defects (cracks).

\subsection{Micro-hardness}

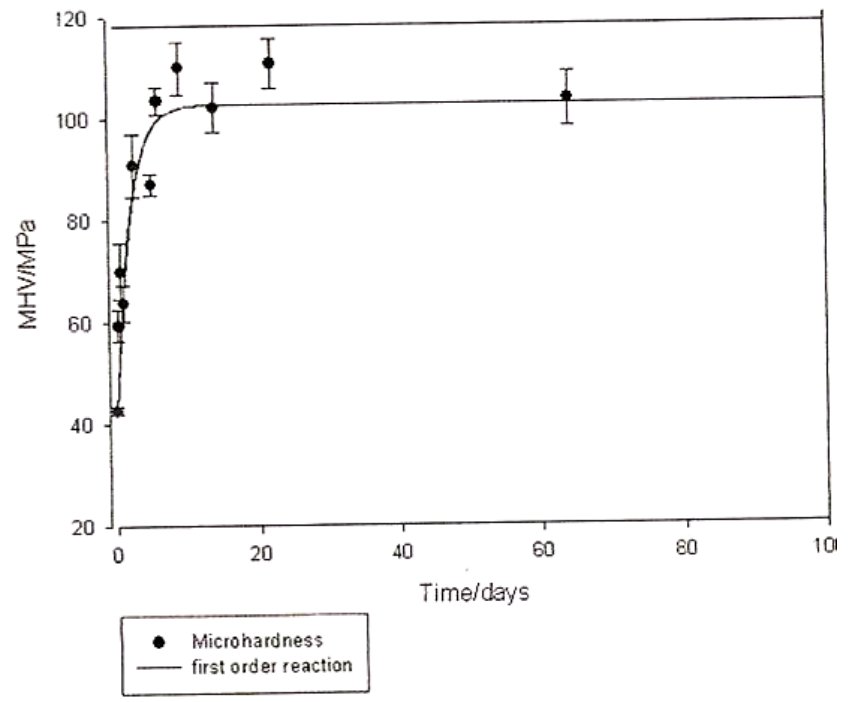

Figure 5. Changes in surface hardness with degradation time for injection molded HDPE samples, correlated with equation for chemical reaction kinetics.

The changes in cutting properties with degradation, as shown in Figure 2 and 3, indicate clearly that mechanical properties are significantly altered by degradation. This can be verified by micro-hardness measurements. Changes in surface hardness with time show the rapid increase and saturation within 20 days (see Figure 5). The measured data can be fitted with equation for the first order chemical reaction:

$$
\mathrm{H}-\mathrm{H}_{\mathrm{o}}=1-\exp (-\mathrm{Kt})
$$


We can plot the front progress by using micro-hardness data obtained from the surface hardness measurements and combine them with the data for the front progress.

The profile of the degradation front can be analyzed by using data for micro hardness testing of the sample surface. This will provide information on the rate of degradation within the surface layer and can help us to understand the observed sharp degradation front, followed by a constant level of degradation (saturation).

The changes in micro-hardness of the surface with degradation time show the saturation of mechanical properties. This must occur also for layers inside the sample in order to follow the progress of the degradation front. This behavior is similar to diffusion process and same equation can be used, based on first order kinetic reaction (equation 3), as reported previously for diffusion of liquids into solids [31, $32,33]$. The time scale can be replaced by the distance scale using equation 2 .

$$
\mathrm{H}-\mathrm{H}_{\mathrm{o}}=1-\exp \left(-(\mathrm{K} / 2 \mathrm{~A})\left(\left(\mathrm{x}_{\mathrm{D}}{ }^{2}-\mathrm{x}^{2}\right)+2 \mathrm{~B}\left(\mathrm{x}_{\mathrm{D}}-\mathrm{x}\right)\right)\right)
$$

where $\mathrm{K}\left(1 /\right.$ day) is the reaction constant, $\mathrm{A}$ (micron ${ }^{2} /$ day) is the constant for degradation front rate, $\mathrm{B}$ (micron) is the rate limiting constant and $\mathrm{x}_{\mathrm{D}}$ (micron) is the degradation front position for a particular time. The micro-hardness data through the sample thickness are shown in Figure 6.

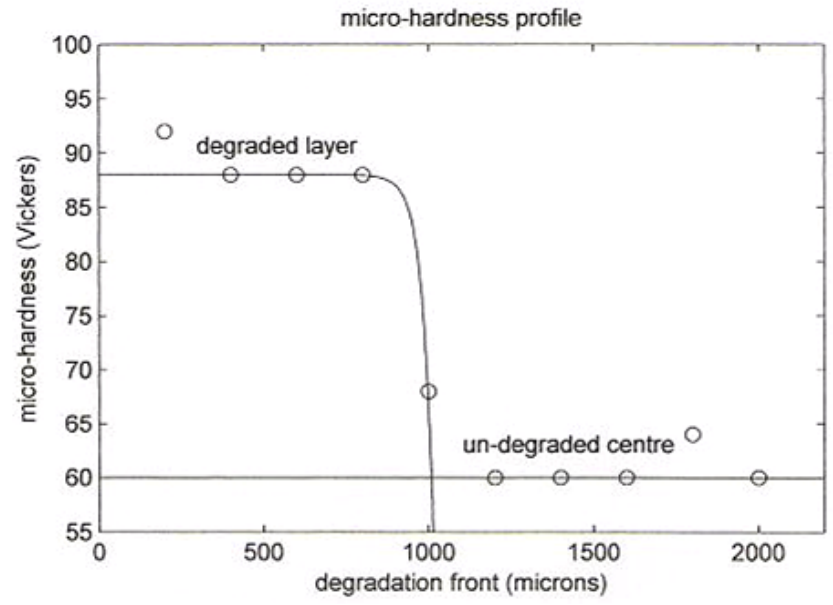

Figure 6. Micro-hardness profile after 14 days degradation. Scatter of data is mainly influenced by surface roughness, resulting from cutting. The experimental data ('o') are averaged for two measurements and compared with equation 4 .

It confirms the saturation of degradation level and steady progress of degradation from the surface to the degradation front as shown by micro-hardness measurements and cutting properties. This provides evidence, that the degraded layer reached saturation and that there is a sharp transition to the un-degraded core of the sample.

\subsection{Carbonyls}

Formation of carbonyls has been extensively studied by FTIR spectroscopy (see e.g. [23-26]). Partially overlapping peaks can be de-convoluted as shown in Figure 7. The proportion of individual peaks remains nearly constant, independent of the degradation time. However, the de-convolution is not unique and is dependent on the selection of the peaks positions.

The measurements of carbonyl profile show clearly that there is an exponential decay of the carbonyl peak, corresponding to Gaussian probability function:

$$
\mathrm{CI}=\mathrm{t} \operatorname{erfc}(\mathrm{x})
$$

Where CI is the carbonyl index, $\mathrm{t}$ is time and $\mathrm{x}$ is the distance from the surface.

The spatial distribution of carbonyls shows that they are concentrated within the surface layer. Data plotted in Figure 8 are compared with 'Carbonyl Index', (ratio of integrated peaks at 1720 and 1492) with equation 5.

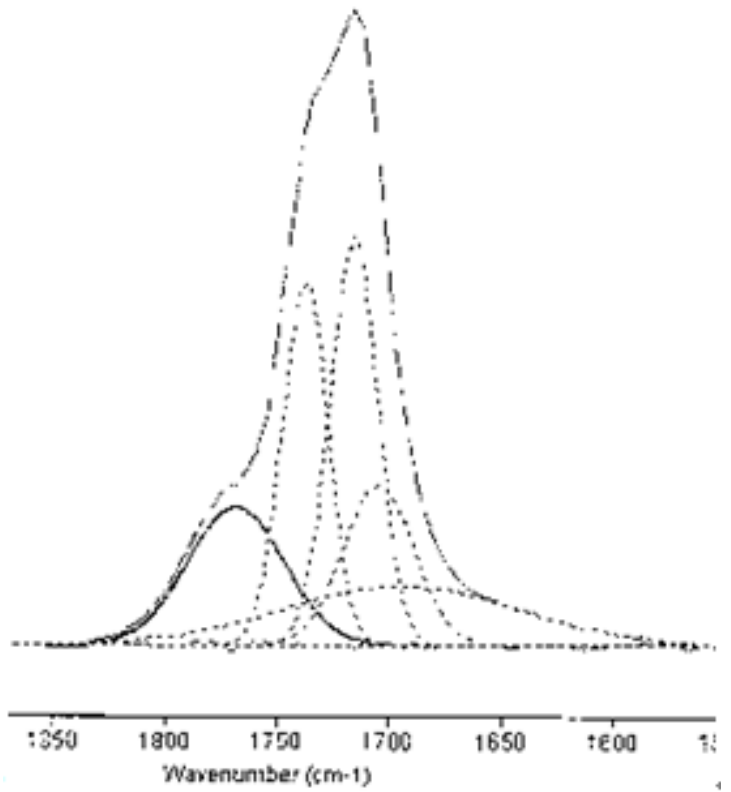

Figure 7. Characteristic FTIR peaks $\left(\mathrm{cm}^{-1}\right)$ de-convoluted using GRAMS 32 program. The peaks are from right to left: at 1690 alkenes, 1700 carboxylic acid, 1714 ketone, 1733 aldehyde and ester, 1780 lactone. Sample degraded for 14 days. The peak area is not related to concentration, but to absorption activity.

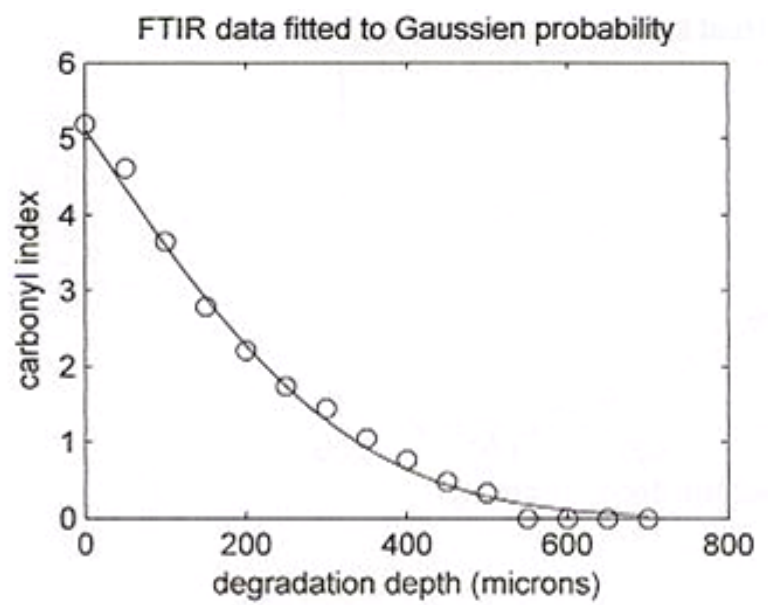

Figure 8. Measured carbonyl index data 'o' after 14 days of degradation at $110^{\circ} \mathrm{C}$, fitted with Gaussian probability function (5). 
On the other hand, the observed degradation front is progressing steadily through the sample thickness, confirming that carbonyl formation is not responsible for changes in mechanical properties (micro-hardness).

The cutting properties however do not correlate with the carbonyl density and indicate together with the micro-hardness measurements and osmium staining intensity, that there is a constant level of degradation throughout the degraded layer, progressing with slowing down speed. This can be interpreted very well with the diffusion theory of Molecular Sorption [31, 32, 33].

\subsection{Surface Defects}

The presence of cracks can be eliminated by re-melting the surface as shown in Figure 9. The cracks are most likely produced by friction of the molten polymer in the cold mould. Cracks are not observed when polymer is compression moulded or in contact with a hot surface (see Figure 9). This formation of cracks can be reduced by additives (e.g. external lubricant) or by temperature of the mould. This will however affect the moulding rate and also the properties of the finished product.

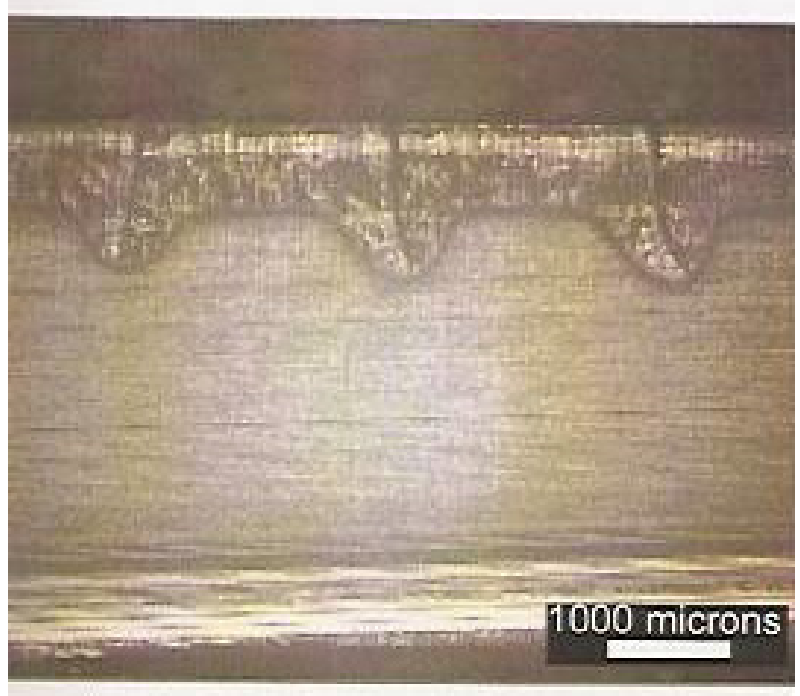

Figure 9. Cross section of a sample which had the bottom surface re-melted on a hot plate. No cracks were observed and the degradation rate is slower.

\subsection{Osmium Staining}

Osmium staining is used to reveal the isolated $\mathrm{C}=\mathrm{C}$ bonds. In conjugated structures the de-localisation of electrons prevents the chemical reaction needed for bonding of osmium tetroxide. However the formation of alkenes can only be carried out by free radicals, which can remove hydrogen atoms from the polymer chain. These radicals can be carried from the surface to the front boundary, using the conductive conjugated polymer back bone. The isolated $\mathrm{C}=\mathrm{C}$ bonds however are still present as demonstrated by staining. This means that there is always a whole range of chemical groups present, but the spatial distribution is uneven. In the sample centre the polymer is not degraded. After exposing the centre to air by cutting, the molecules of $\mathrm{OsO}_{4}$, which are not chemically bonded, will quickly evaporate due to their high volatility. Figures 10 and 11 show clearly an even contrast, resulting from a saturated concentration of bonded molecules.

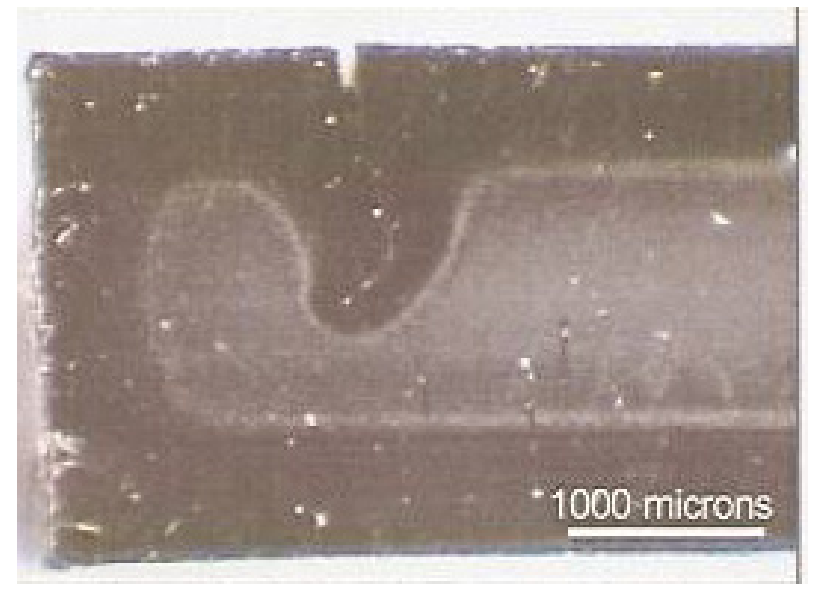

Figure 10. Osmium stained sample degraded for 20 days. Picture obtained in reflection.

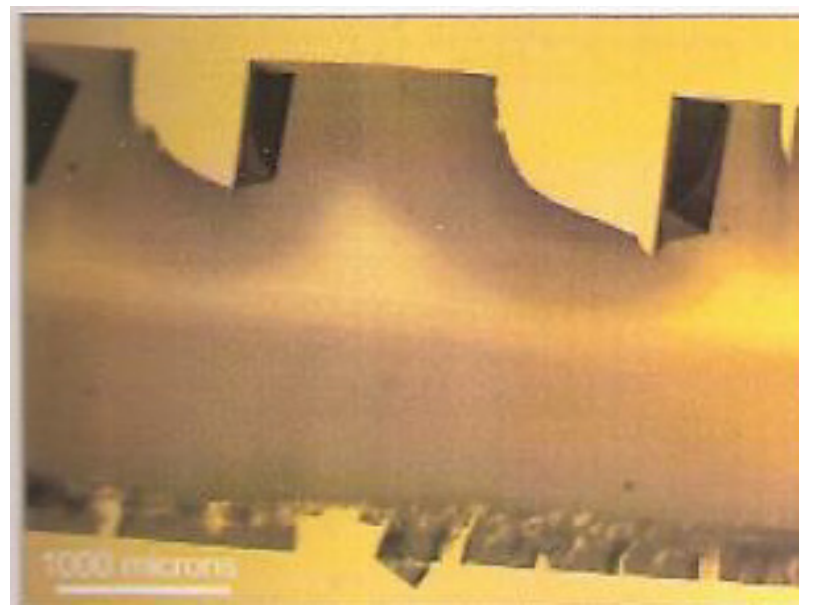

Figure 11. Osmium stained sample degraded for 26 days. Cut section after staining. Presence of crack is revealed by cutting. The degradation rate is clearly influenced by the crack density.

\section{Discussion}

The experimental results above show two independent mechanisms of degradation: oxidation of the surface layer by formation of carbonyls (as revealed by FTIR) and a continuous progress of the degradation front (as revealed by cutting properties, osmium tetroxide staining and micro hardness). These two mechanisms of degradation seem to be independent. The concentration of carbonyls in the surface layer can only be explained by formation of free radicals which cannot move in un-degraded non-conductive layer and thus take two hydrogens from the same carbon atom and 
replace them with oxygen. The probability of carbonyl formation is dependent on the presence of oxygen close to the reaction site and on ionization probability by light radiation. This means that the depth of degradation layer remains constant and is dependent on the probability of photon reaching that depth. This probability is dependent on the mean free path and can be interpreted with Gaussian error function, where time dependent concentration is the variable but not the distance, as the photon depth of penetration stays the same. This can be calculated using the equation 4 and 5 and the results are plotted in Figure 5, 6 and 9. The correlation with the experiment is very good. The differences between micro-hardness measurements and carbonyl profile are significant (see Figure 12).

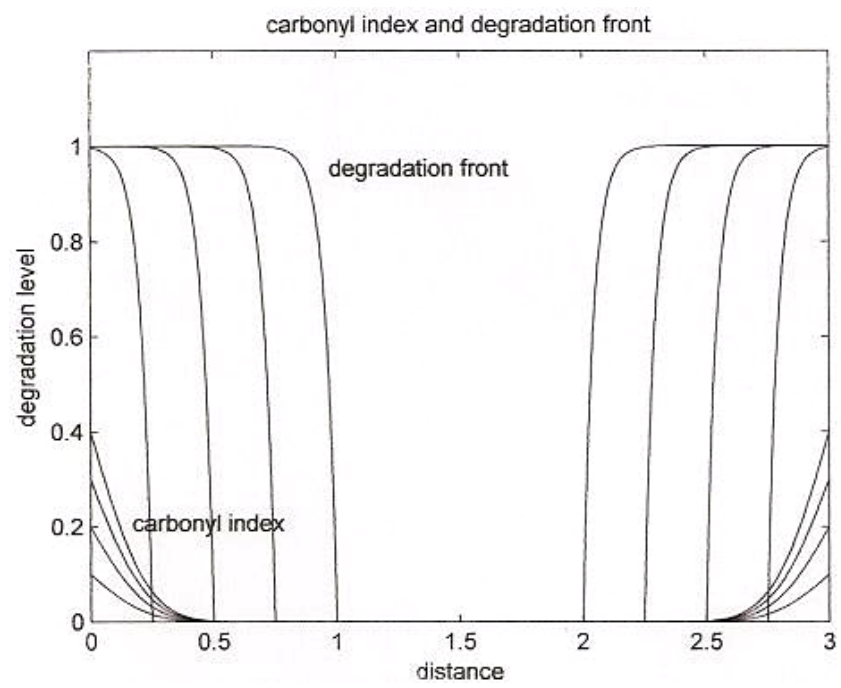

Figure 12. Calculated graphs using FTIR and micro-hardness data and equations 2 and 4.

It might be obvious that the cutting properties do not correlate with the carbonyl density and indicate together with the micro-hardness measurements and osmium staining intensity, that there is a constant level of degradation throughout the degraded layer, progressing with slowing down speed. This can be interpreted very well with the diffusion theory of Molecular Sorption [31, 32, 33].

This would require an assumption that within the surface layer free radicals are formed by the photons and in the presence of oxygen, forming carbonyls. The density of these groups formed is dependent on the intensity of light, which is diminishing with the depth. It is not possible to explain the carbonyl profile by the concentration of oxygen, as this would require diffusion into the centre and not the increase of carbonyls in the surface layer.

It might be more difficult to explain the formation of degraded layer, which propagates into the sample, with sharp front, leaving behind polymer degraded to a constant level. This behavior is similar to diffusion mechanism of Molecular Sorption, but it would be necessary to assume that there is a saturation level which is achieved quickly by chemical kinetic reaction at the diffusion front, and that the structure formed is stable. It would also be necessary to assume that free radicals are formed at the surface and diffuse to the degradation front through the degraded polymer. This might be possible, as the degraded polymers can be highly conductive (see [34] describing the increase in conductivity by seven orders in electron beam irradiated polyvinylchloride).

\section{Conclusions}

This work shows that the degradation process is controlled by changes in the surface layer, and propagates with time to the centre of the sample. The chemical nature of this degraded layer is not fully understood, but osmium staining indicates that formation of alkenes is one of the major contributors to the loss of mechanical properties. The degradation also opens the pre-existing cracks, which are formed by the injection molding process.

The propagation of the degraded layer can be best described by the diffusion equation, which requires sharp diffusion front followed by a saturated degradation level. This is an equivalent to saturated concentration dictated by phase diagrams (as observed in systems of polymer/polymer, polymer/solvent, liquid/liquid, metal/metal etc.).

Investigation of the surface properties, like micro-hardness or staining can be used to explain the progress of the degradation front. The free radicals can diffuse through a degraded layer, which is conductive to charged radicals, forming at the surface. This hypothesis is currently the most attractive for explanation of the experimental results reported in this work, but further work is needed, particularly for better understanding of chemical composition of the degraded layer and mobile radicals.

\section{REFERENCES}

[1] U. W. Gedde et al.: Long term properties of hot water polyolefin pipes. Polymer Engineering and Science (1994) Vol. 34(24) p. 1773-1787

[2] L. Reich and S. S. Stivala: Elements of polymer degradation. (1973) McGraw-Hill,N. Y.

[3] D. J. Carlsson and D.M.Wiles: Surface changes during photooxidation of polypropylene. Journal of Materials Science (1970) Vol. 8, p. 419

[4] L. C. I. Struik, Physical aging in amorphous polymers and other materials, Elsevier (1978)

[5] N. C. Billingham and P. D. Calvert, The physical chemistry of oxidation and stabilization of polyolefins, Developments in polymer degradation (1980) Vol. 3, p.139

[6] G. Scott, Mechanisms of polymer degradation and stabilization, Elsevier (1990)

[7] L. Reich and S. S. Stivala, Elements of polymer degradation, McGraw-Hill, N.Y. (1973) 
[8] G. D. Smith, K. Karlsson and U. W. Gedde, Polymer engineering and science (1959), Vol. 41,

[9] F. Gugumus, Thermooxidative degradation of polyolefins in the solid state, Experimental kinetics of functional group formation, Polymer degradation and stability (1996) Vol. 52, p.131-144

[10] F. Gugumus, Photooxidation of polymers and its inhibition: Oxidation inhibition in organic materials, (editors J. Pospisil and P. Klemchuk), CRC Press (1990) p. 29-161

[11] F. Gugumus, Re-examination of the thermal oxidation reactions of polymers 1: New views of an old reaction: Polymer degradation and stability (2001) Vol. 74, p. 327-339

[12] F. Gugumus, Re-examination of the thermal oxidation reactions of polymers 2: Thermal oxidation of polyethylene, Polymer degradation and stability (2002), Vol. 76, p. 329-340

[13] F. Gugumus, Thermooxidative degradation of polyolefins in the solid state, Part 5 Kinetics of functional group formation in PE-HD and PE-LLD: Polymer degradation and stability (1997), Vol. 55, p. 21-43

[14] T. Corales et al. Photooxidative and thermal degradation of polyethylenes: Interrelationship by chemiluminescence, thermal gravimetric analysis and FTIR data. Journal of photochemistry and photobiology A, (2002), Vol. 147, p. 213-224

[15] D. J. Carlson and D. M. Wiles, Surface changes during photooxidation of polypropylene: Journal of materials science (1970) Vol. 8. (polymer letters) p. 419

[16] Y. Hori, Organic peroxy radicals in polymeric systems in Peroxyl Radicals (ed. Alfassi) John Wiley \& sons, (1997), p. 355-406

[17] P. Gijsman G. Maijers and G. Vitarelli, Comparison of the UV degradation chemistry of polypropylene, polyethylene polyamide 6 and polybutylene terephthalate. Polymer degradation and stability (1999) Vol. 65, p. 433-441

[18] V. G. Burkhudaryan, Alterations of molecular characteristics of polyethylene under the influence of UV radiation: Polymer degradation and stability (2000) Vol. 41, p. 5787-5791

[19] F. Gugumus, Thermolysis of polyethylene hydroperoxides in the melt 2: Formal kinetics of hydroperoxide decomposition. Polymer degradation and stability (2000) Vol. 69, p. 30-35

[20] A. W. Lee, J. P. Santerre and E. Boynton, Analysis of released products from oxidized ultra-high molecular weight polyethylene incubated with hydrogen peroxide and salt solutions. Biomaterials (2000), Vol. 21, p. 851-861
[21] C. Naddeo et al. Mechanical and transport properties of irradiated linear low density polyethylene (LLDPE). Polymer degradation and stability (2001) Vol. 72, p.239-247

[22] R. Setnescu, S. Jipa and Z. Osawa. Chemiluminescence study on the oxidation of several polyolefins I: Thermal induced degradation of additive free polyolefins: Polymer degradation and stability (1998) Vol. 60, p. 377-383

[23] J. M. Chalmers and N. J. Everall, Vibrational spectroscopy: Polymer characterization (ed. Hunt and James) (1993), Blackie Academic and professional, p. 69-106

[24] G. A. George et al. Real time analysis of the thermal oxidation of polyolefins by FT-IR emission: Polymer degradation and stability (1995) Vol. 48, p. 199-210

[25] W. Klopffer. Introduction to polymer spectroscopy: Polymer properties and applications (1984) Springer Verlag

[26] John D. Roberts and Marjorie C. Caserio: Basic Principles of Organic Chemistry, Addison Wesley (1979)

[27] G. Owen and D. Vesely: An easy and economical method for staining electron microscopy specimens with osmium tetroxide vapour. Proceedings RMS, Vol. 20-6, November 1985

[28] M. A. Parker and D. Vesely: Contrast enhancement and polymer identification in the electron microscope by the formation and staining of unsaturated double bonds.Microscopy Research and Techniques, (1993) Vol. 24, p. $333-339$

[29] Daniel S. Kemp and Frank Vellaccio: Organic Chemistry, Worth Publishers (1980)

[30] G. Zanfirova and A. Dimitrova: Some methodical contributions to the Vickers microhardnes technique. Polymer testing (2000) Vol.19 p.533-542

[31] D.Vesely: Molecular sorption mechanism of solvent diffusion in polymers.Polymer (2001) Vol. 42, p. 4417-4423

[32] D. Vesely Diffusion of liquids in polymers.International Materials Reviews (2008) Vol. 53, p. 299-315

[33] D. Vesely and Ming Zhu: The effect of surface conditions on diffusion and permeability of acetone through uPVC. Universal Journal of Materials Science (2014) Vol. 2(5), p. 96-104

[34] D. Vesely, D. Finch and G. Cooley: Electrical properties of polymers modified by electron beam irradiation. Polymer (1988) Vol. 29 p. 1402-1406 